\title{
Research on network management system structure of wireless sensor Jianjun Zhang ${ }^{1, a}$
${ }^{1}$ Department of Computer Science, Gansu Normal University for Nationalities, Hezuo Gansu China \\ azhangjj690304@126.com
}

Keywords: wireless sensor network; management; system structure

\begin{abstract}
Network management is a way of network management, the network can be properly efficient operation. Its purpose is to make the network resources more effectively use. Wireless sensor network management refers to the process control of a complex sensor network so that it has the highest control ability and the ability to perceive. This article mainly conducts the research to the management system structure framework for wireless sensor networks.
\end{abstract}

\section{Introduction}

Considering the content and the sensor network protocol stack for network management, has the following two-dimensional structure, namely horizontal layer communication protocol and sensor network management longitudinal plane [1], as shown in figure 1. Communication protocol layer can be divided into physical layer, link layer, network layer, transport layer,application layer, and network management aspects can be divided into energy management,fault management and task management plane. The existing management is mainly used to coordinate the function of different levels, in order to obtain the optimal design considering the energy consumption management, fault management and configuration management.

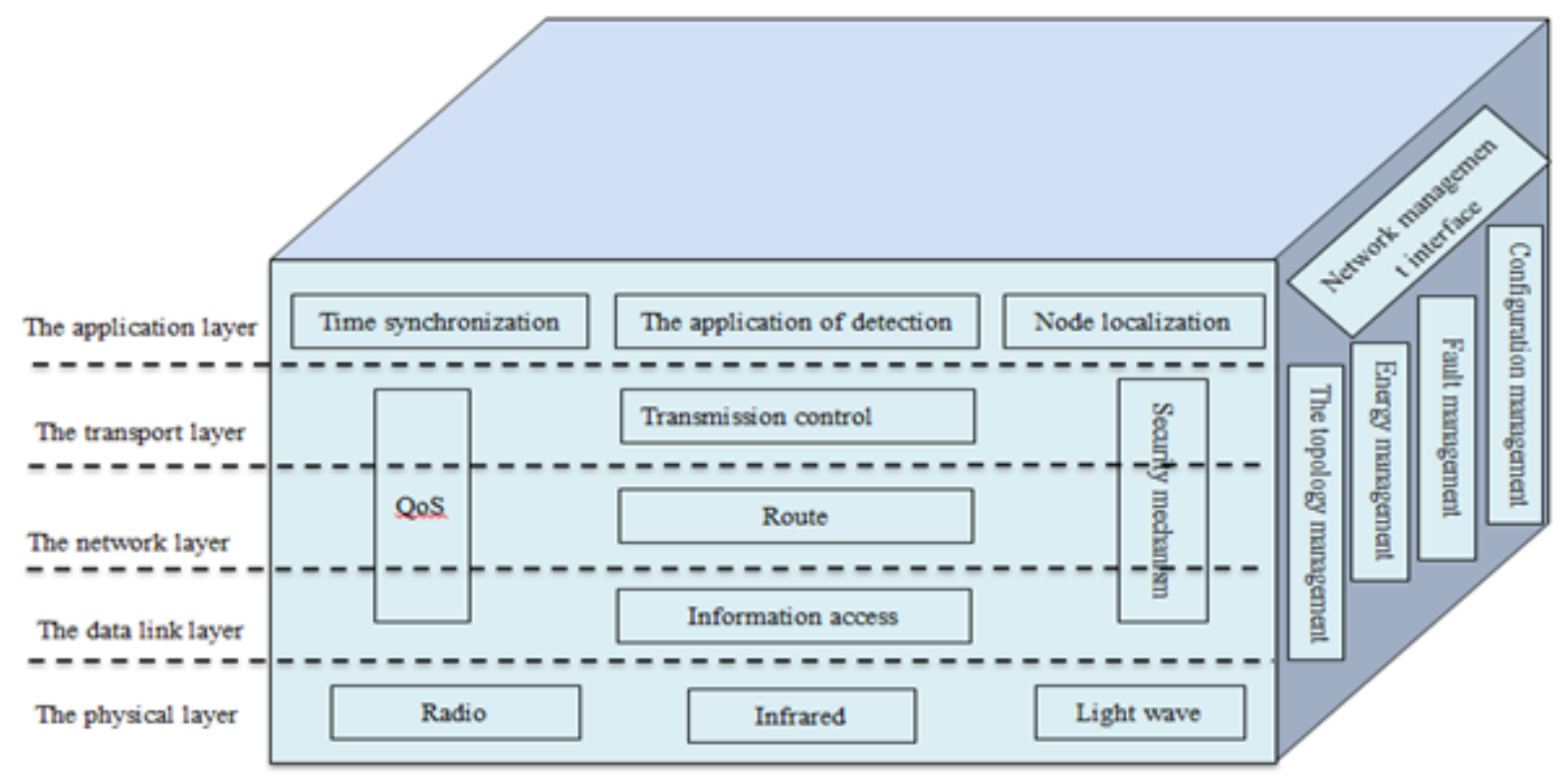

Figure 1 two dimensional diagram of sensor network

Sensor network management although can be divided into the above aspects, but the above are not isolated, they in network management system are mutual cooperation to complete the network management, to make the sensor network monitoring application function maximization.

So far, we have two kinds of wireless sensor network management architecture: one is the Linnyer Beatrys proposed by Ruiz and MANNA wireless sensor network management architecture[2-3]; the other is based on the distributed wireless sensor network management agent framework proposed by Feng and Wang[4]. 


\section{MANNA sensor network management architecture}

Linnyer Beatrys Ruiz proposed MANNA management architecture for wireless sensor networks[5].MANNA management architecture consists of three systems, respectively is: the function of system, information system and physical system.

\section{The function system}

The function of network management system management system described in, Agent and management information base[6] function of the relationship between the three. As wireless sensor network, different distributions may have multiple nodes and node, Beatrys Ruiz proposed the Linnyer manager and agent according to the need of practical application, should have the function and the position in network management system. For the establishment of management information base and its relationship with Agent is often the one correspondence, so its distribution is similar to Agent.

\section{Information system}

The MANNA information system mainly is the information model based on object oriented. Basically speaking, the system includes two modules, these two types of modules are in accordance with the interaction between the Agent and Manager information to design. Information model provides between the management of resources and object-oriented class image and matching. Information model contains represents is manageable resources at different levels of abstraction of the class, the level of abstraction contains abstract, management level of abstraction and the function of the network performance level of abstraction, it is for realizing towards standardizing the most critical step design and sensor network management system.

\section{The physics system}

The physical system is to achieve the function of system design. In the physical system design of network management protocol, physical location, node function, the need to achieve the service and support for the WSN interface needs to be defined. In the management entity between the interface must be lightweight part, MANNA does not contain this part, but it is a very important part of the management. In the current network management protocol such as SNMP[7], CMIP, WBM and ANMP are not in the sensor network design, compared to these general network, has its unique local sensor network. But also for the sensor network management involves many aspects of the system, the need to take into account the sensor node hardware problems, the physical location and function, the implementation of the management service and support interface.

\section{Network management architecture of distributed sensor network based on agent}

Wang Feng put forward the management framework of distributed sensor network proxy based on [4]. Management system is composed of sensor subsystem, data fusion sub system, decision support subsystem and network management subsystem, the whole network is a closed loop system. The sensor system is composed of sensor nodes, data fusion sub system task is to evaluate and predict the target state and status, analysis and Research on the condition assessment and risk assessment decision support system of the results, a network management system to control the sensor based on decision support system of the proposed activities to enhance the monitoring performance of sensor networks, such as shown in figure 2. 


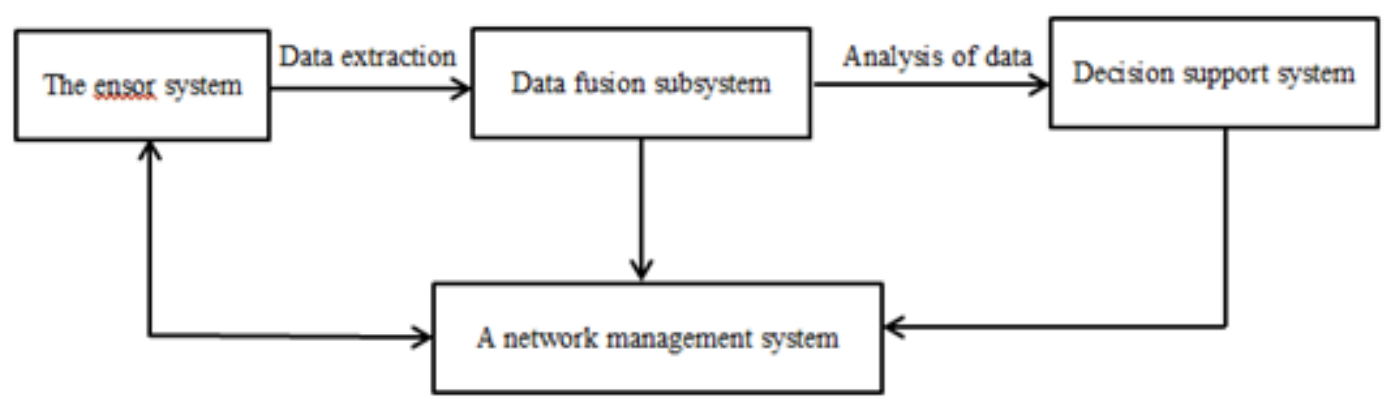

Figure 2 based on the management system of distributed sensor network proxy

Wang Feng thinks that the sensor network management is in according to the current networkstate, looking for a pair of nodes in the network the optimal configuration and management scheme, and the system is configured so that each performance index of the optimal.

In order to solve the management problems of hierarchical sensor networks, Wang Feng summarizes the shortcomings of client / server network management based on the model, proposes a new pattern is the mobile agent model management, as shown in figure 3.

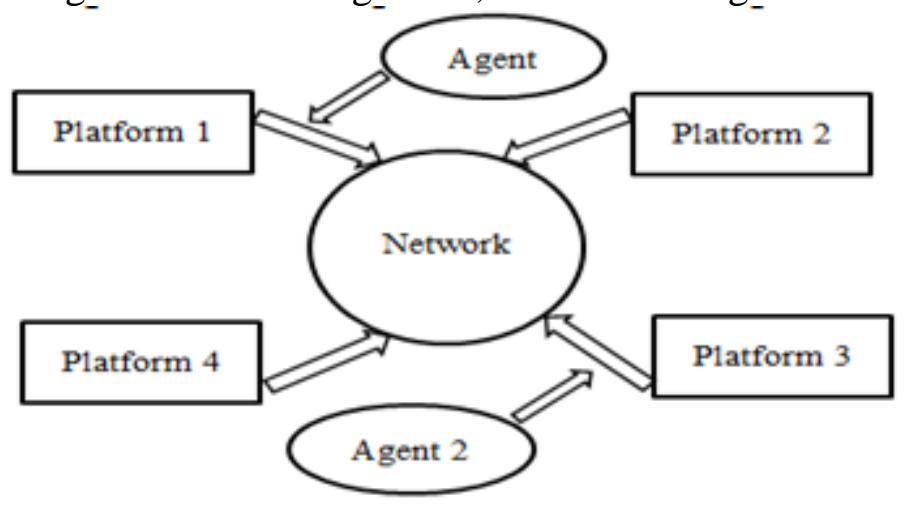

Figure 3 mobile agent management model

Mobile Intelligent Agent-based DSN by using a new model: data retention in the local, data fusion is transferred to the remote. In this model, the intelligent agent is used for broadcasting network platform data. Some special intelligent agent service for data fusion, the implementation of information fusion or resource allocation rules task. Compared with the client / server mode, this model has the following advantages: first, because the agent to transmit only very small capacity, greatly reducing the network bandwidth; second, the mobile agent based network is scalable, so the number of sensors does not affect the network performance; third, when the network connectivity, mobile agent can be transmitted, when there is a fault in the network and then reconnected, MIADSN is not affected by the network reliability.

Mobile agent is a kind of software, its function and role and Agent is similar, the results need to rely on the network management agent output. The use of mobile agent to design sensor network management has two kinds: one is the design idea of each Agent to a task execution in network management; another is the existence of a number of Agent system, each Agent determine the polling among several sensor nodes. The sensor node management into the agent, a capable of several nodes management agent. Since the agent is usually very small software code, generally will not cause large energy consumption in the system when the transmission.

\section{Author brief introduction}

Jianjun Zhang (1969-), male, associate professor, Gansu Yuzhong people, mainly engaged in computer specialized teaching and research work. 


\section{References}

[1] Estrin D. Tutorial "Wireless Sensor Networks" Part IV: Sensor Network Protocols[M], MobiCom, 2002

[2 Linnyer Beatrys Ruiz, Jose Marcos Nogueira, Antonio A. F Loureiro, MANNA. A Management Architecture for Wireless Sensor Networks[J], IEEE Communications Magazine, volume. 41, Issue. 2, February 2003:116-125.

[3] Ruiz LB, Silva FA, Braga T.R.M, Nogueira LM.S, Loureiro, A.A.F. On Impact of Management in Wireless Sensors Networks, Network Operations and Management Symposium[J], 2004. NOMS 2004. IEEE/IFIP, volume.1,19-23 April 2004: 657-670.

[4] Wang Feng, Tian Qichuan, Gao Quanxue, Pan Quan, A Study of Sensor Management Based on Sensor Networks[C], Proceedings of the 2003 IEEE International Conference on Robotics, Intelligent Systems and Signal Processing. 2003 IEEE International Conference, volume.2,8-13,October 2003:1058-1062.

[5] Ruiz, L.B. MANNA: Uma Arquitetura para o Gerenciamento de Redes de Sensores Sem Fio[J]. Tese de doutorado, Universidade Federal de Minas Gerais. 2003

[6] Yu Hai bin, Ceng Peng, Wang Zhong feng. Research on distributed communication protocol for wireless sensor networks [J]. Journal of China Institute of communications, 2004,25 (10):102-110

[7] Li Mingjiang SNMP simple network management protocol [M] Beijing: Publishing House of electronics industry, 2007 